\title{
Simulation study of beam-beam effects
}

\author{
Yuan Zhang, ${ }^{1}$ Kazuhito Ohmi, ${ }^{2}$ and Limin Chen ${ }^{1}$ \\ ${ }^{1}$ Institute of High Energy Physics, Yuquan Road 19(B), Beijing 100049, China \\ ${ }^{2}$ KEK, 1-1 Oho, Tsukuba, 305-0801, Japan \\ (Received 19 May 2005; published 26 July 2005)
}

\begin{abstract}
The Beijing Electron-Positron Collider (BEPC) will be upgraded to BEPCII, and the luminosity will be about 100 times higher. We developed a three-dimensional strong-strong PIC code to study the beambeam effects in BEPCII. The transportation through the arc is the same as that in Hirata's weak-strong code. The beam-beam force is computed directly by solving the Poisson equation using the Fourier analysis and cyclic reduction method, and the boundary potential is computed by circular convolution. The finite bunch length effect is included by longitudinal slices. An interpolation scheme is used to reduce the required slice number in simulations. The standard message passing interface is used to parallelize the code. The computing time increases linearly with $(n+1)$, where $n$ is the slice number. The calculated luminosity of BEPCII at the design working point is $\sim 50 \%$ of the design value. The best area in the tune space is near $(0.505,0.57)$ according to the survey, where the luminosity is $\sim 80 \%$ of the design.
\end{abstract}

DOI: 10.1103/PhysRevSTAB.8.074402

PACS numbers: 29.20.Dh, 29.27.- a, 41.75.Ht

\section{INTRODUCTION}

The Beijing Electron-Positron Collider (BEPC) was constructed for both high energy physics and synchrotron radiation (SR) researches. As a unique $e^{+} e^{-}$collider operating in the $\tau$-charm region and the first SR source in China, the machine has been well operated for over 16 years since it was put into operation in 1989.

BEPCII is an upgrade scheme from BEPC. It is a double ring machine. Following the success of the KEK B factory (KEKB), the crossing scheme was adopted in BEPCII, where two beams collide with a horizontal crossing angle of $2 \times 11 \mathrm{mrad}$. The design luminosity of BEPCII is $1.0 \times$ $10^{33} \mathrm{~cm}^{-2} \mathrm{~s}^{-1}$ at $1.89 \mathrm{GeV}$, which is about 100 times higher than BEPC [1].

The beam-beam interaction is one of the most important limiting factors to determine the luminosity of storage ring colliders. Because of the complexity of the interaction, computer simulations are necessary to study it quantitatively. There have been various types of computer codes on this topic, such as weak-strong simulation [2] and strongstrong simulation [3-7]. Historically, the weak-strong simulation, which is not self-consistent, has been employed in order to simulate the effect in a reasonable computing time. The strong-strong simulation, which requires large amounts of computer resources, has recently become feasible due to the fast progress in computing power. Now the two-dimensional simulation without finite bunch length effect can be done using a personal computer in a reasonable time, while a supercomputer is still necessary in the three-dimensional strong-strong simulation. The strong-strong codes now have a reliable predictive capability of realistic beam-beam interaction and the simulation results show a good quantitative agreement with the experimental observations [8].
In order to include the crossing angle effects, the beambeam simulation needs to take the bunch length into account. We have developed a new three-dimensional strongstrong particle-in-cell (PIC) code SBBE, which was based on our previous work [9]. The code was written in standard $\mathrm{C}$ language and parallelized with the standard message passing interface (MPI). The model and algorithm used in the code are explained in Sec. II. We have studied the beam-beam effects in BEPCII using SBBE, and the results are presented in Sec. III.

\section{MODEL AND ALGORITHM}

The two colliding beams are both represented by macroparticles in SBBE. A macroparticle is treated as a single electron or positron dynamically. We initialize the macroparticles with the six-dimensional Gaussian distribution according to the optics parameters at the interaction point (IP) and the nominal emittance of the beam.

The one-turn map of macroparticles consists the following two parts:

(1) Beam-beam interaction near IP. The bunch length effect is included by longitudinal slices. A slice interacts with the opposite slices one by one. When the interaction between two slices is considered, the potentials at two longitudinal points generated by a slice are computed directly and, respectively, by solving the Poisson equation. The potentials between the two points are calculated with linear interpolation. The macroparticles in a slice are kicked by the opposite slices, and drift between two collision points near IP.

(2) Transportation through the arc. Single particle dynamics in three-dimensional space is taken into account. We only consider the linear map with synchrotron radiation. 
In the following, the model and algorithm used in the code are described in detail.

\section{A. Transportation through arc}

We employ the so-called accelerator coordinate $\mathbf{x}=$ $\left(x, p_{x}, y, p_{y}, z, p_{z}\right)$ to describe the motion of particles. Here $x$ and $y$ are horizontal and vertical coordinates, respectively, while $p_{x}$ and $p_{y}$ are the corresponding transverse momenta normalized by the total momentum $\left(P_{0}=\right.$ $E_{0} / c$ ) of a reference particle. $z$ is the delay time and defined as $z=s-\operatorname{ct}(s)$, where $t$ is the arrival time at the position $s$, and $p_{z}=\left(P-P_{0}\right) / P_{0}$ is the momentum deviation from the nominal.

Following Hirata's BBC code [10], the transportation through the arc consists of the following maps:

\section{From accelerator coordinates to normalized coordi- nates}

The transformation from accelerator variable $\mathbf{x}$ to normalized variable $\mathbf{X}$ can be written as

$$
\mathbf{X}=B R H \mathbf{x}
$$

The dispersion matrix $H$ in Eq. (1) is characterized by the transverse dispersion functions $D_{x}, D_{p x}, D_{y}$, and $D_{p y}$ at IP,

$$
H=\left(\begin{array}{ccc}
I & 0 & -H_{x} \\
0 & I & -H_{y} \\
-J_{2} H_{x}^{t} J_{2} & -J_{2} H_{y}^{t} J_{2} & I
\end{array}\right)
$$

where

$$
H_{x}=\left(\begin{array}{cc}
0 & D_{x} \\
0 & D_{p x}
\end{array}\right), \quad H_{y}=\left(\begin{array}{cc}
0 & D_{y} \\
0 & D_{p y}
\end{array}\right),
$$

and $J_{2}$ is the $2 \times 2$ symplectic matrix

$$
J_{2}=\left(\begin{array}{cc}
0 & 1 \\
-1 & 0
\end{array}\right)
$$

The Teng matrix $R$ in Eq. (1) is defined as

$$
R=\left(\begin{array}{ccc}
b I & J_{2} R_{2}^{t} J_{2} & 0 \\
R_{2} & b I & 0 \\
0 & 0 & I
\end{array}\right)
$$

where

$$
b=\sqrt{1-\left|R_{2}\right|}, \quad R_{2}=\left(\begin{array}{ll}
r_{11} & r_{12} \\
r_{21} & r_{22}
\end{array}\right) .
$$

The Twiss matrix $B$ in Eq. (1) is defined as $\operatorname{diag}\left(b_{u}, b_{v}, b_{w}\right)$ and each $b_{i}(i=u, v, w)$ is a $2 \times 2$ matrix

$$
b_{i}=\left(\begin{array}{cc}
\frac{1}{\sqrt{\beta_{i}}} & 0 \\
\frac{\alpha_{i}}{\sqrt{\beta_{i}}} & \sqrt{\beta_{i}}
\end{array}\right),
$$

where $\alpha_{i}(i=u, v)$ and $\beta_{i}(i=u, v)$ are the Twiss parame- ters at IP. In our code, $\alpha_{w}$ is assumed to be zero and $\beta_{w}$ is calculated as $\sigma_{z} / \sigma_{e}$, where $\sigma_{z}$ is the bunch length and $\sigma_{e}$ is the relative energy spread.

\section{Transportation with synchrotron radiation}

With synchrotron radiation, the arc transportation in the normalized coordinates is

$$
\begin{array}{r}
\left(\begin{array}{l}
\mathbf{X}_{1} \\
\mathbf{X}_{2}
\end{array}\right) \rightarrow \lambda_{u} m_{u}\left(\begin{array}{l}
\mathbf{X}_{1} \\
\mathbf{X}_{2}
\end{array}\right)+\sqrt{\epsilon_{x}\left(1-\lambda_{u}^{2}\right)}\left(\begin{array}{l}
\hat{r}_{1} \\
\hat{r}_{2}
\end{array}\right), \\
\left(\begin{array}{l}
\mathbf{X}_{3} \\
\mathbf{X}_{4}
\end{array}\right) \rightarrow \lambda_{v} m_{v}\left(\begin{array}{l}
\mathbf{X}_{3} \\
\mathbf{X}_{4}
\end{array}\right)+\sqrt{\epsilon_{y}\left(1-\lambda_{v}^{2}\right)}\left(\begin{array}{c}
\hat{r}_{3} \\
\hat{r}_{4}
\end{array}\right), \\
\left(\begin{array}{c}
\mathbf{X}_{5} \\
\mathbf{X}_{6}
\end{array}\right) \rightarrow\left(\begin{array}{cc}
1 & 0 \\
0 & \lambda_{w}^{2}
\end{array}\right) m_{w}\left(\begin{array}{l}
\mathbf{X}_{5} \\
\mathbf{X}_{6}
\end{array}\right)+\left(\sqrt{\sqrt{\epsilon_{z}\left(1-\lambda_{w}^{4}\right)} \hat{r}_{5}}\right) .
\end{array}
$$

Here $\hat{r}$ 's are independent Gaussian random variables with unit variance, $\lambda_{i}=\exp \left(-1 / T_{i}\right)$ with $T_{i}$ the damping time in unit of the number of turns, and $\epsilon_{z}$ is calculated as $\sigma_{z} \sigma_{e}$.

\section{From normalized coordinates to accelerator coordi- nates}

The transformation from normalized variable $\mathbf{X}$ to accelerator variable $\mathbf{x}$ is

$$
\mathbf{x}=H^{-1} R^{-1} B^{-1} \mathbf{X} .
$$

\section{B. Field solver}

For head-on colliding beams, the beam-beam force is nearly transverse and there exists interaction if and only if a particle meets another in the opposite beam. The bunch (or slice) is treated approximately as a two-dimensional distribution when the beam-beam force is considered. Given a charge distribution $\rho(x, y)$, the potential $\phi(x, y)$ generated by the beam satisfies the Poisson equation

$$
\left(\frac{\partial^{2}}{\partial x^{2}}+\frac{\partial^{2}}{\partial y^{2}}\right) \phi(x, y)=-\frac{\rho(x, y)}{\epsilon_{0}} .
$$

The beam-beam force is evaluated from the potential

$$
\delta p_{x}=-\frac{e}{P_{0} c} \frac{\partial \phi(x, y)}{\partial x}, \quad \delta p_{y}=-\frac{e}{P_{0} c} \frac{\partial \phi(x, y)}{\partial y},
$$

where $P_{0}$ is the nominal momentum of the beam that experiences the force. The solution of the potential $\phi$ can be expressed as

$$
\phi(x, y)=\frac{1}{2 \pi \epsilon_{0}} \int d x^{\prime} d y^{\prime} G\left(x-x^{\prime}, y-y^{\prime}\right) \rho\left(x^{\prime}, y^{\prime}\right),
$$

where $G(x, y)$ is the Green's function

$$
G(x, y)=-\frac{1}{2} \ln \left[x^{2}+y^{2}\right] .
$$


The transverse dimension of the beam is generally much smaller than that of the pipe near IP. Therefore the open boundary condition is assumed.

Cai's method [7] is used to solve the beam-beam field. In order to compute the field generated by a beam, we first deposit macroparticles' charge onto grid points of a twodimensional mesh. The mesh is chosen to be large enough to cover the core of two colliding beams. The triangularshaped cloud (TSC) method is employed for the charge assignment, where the charge of each macroparticle is assigned to its nine nearest points by weight. This scheme ensures conservation of charge.

The boundary potential can be calculated directly by Eq. (14). A fast-Fourier-transform (FFT) method is used to compute them in SBBE. We represent the potential $\phi(x, y)$ by its values at discrete set of points

$$
\begin{aligned}
& x_{j}=x_{0}+j h_{x} \quad(j=0,1, \ldots, J), \\
& y_{l}=y_{0}+l h_{y} \quad(l=0,1, \ldots, L),
\end{aligned}
$$

where $h_{x}$ and $h_{y}$ are horizontal and vertical grid spacing, respectively. We write $\phi_{j, l}$ for $\phi\left(x_{j}, y_{l}\right)$. The points where $j=0, j=J, l=0$ or $l=L$ are boundary points. Here we describe the method by introducing how to compute the potential $\phi_{0, l}$, where $l=0, \ldots, L-1$. Following Eq. (14), $\phi_{0, l}$ can be written as

$$
\begin{aligned}
\phi_{0, l} & =\frac{1}{2 \pi \epsilon_{0}} \sum_{m=1}^{J-1} \sum_{n=1}^{L-1} C_{m, n} G_{m,|l-n|} \\
& =\frac{1}{2 \pi \epsilon_{0}} \sum_{m=1}^{J-1} \phi_{0, l}^{m} \quad(l=0, \ldots, L-1),
\end{aligned}
$$

where $C_{m, n}$ is the charge on point $\left(x_{m}, y_{n}\right), G_{m, n}$ is the value of Green's function $G\left(m h_{x}, n h_{y}\right)$, and $\phi_{0, l}^{m}$ is defined as

$$
\phi_{0, l}^{m}=\sum_{n=1}^{L-1} C_{m, n} G_{m,|l-n|} \quad(l=0, \ldots, L-1) .
$$

$C_{m, n}$ and $G_{m, n}$ are extended, respectively, as

$$
\begin{gathered}
C_{m, n}=0 \quad(L \leq n \leq 2 L-1), \\
G_{m, n}= \begin{cases}\text { arbitrary } & (n=L), \\
G_{m, 2 L-n} & (L<n \leq 2 L-1) .\end{cases}
\end{gathered}
$$

With the extended series, $\phi_{0, l}^{m}$ can be rewritten as a formalism of circular convolution

$$
\phi_{0, l}^{m}=C_{m, l} \otimes G_{m, l} \quad(l=0, \ldots, 2 L-1) .
$$

The value of $\phi_{0, l}^{m}$ in Eq. (21) is equal to that in Eq. (18) for $0 \leq l \leq L-1$, and meaningless for $L \leq l \leq 2 L-1$. The discrete Fourier transforms (DFT) of $C_{m, l}$ and $G_{m, l}$ are evaluated as

$$
\begin{aligned}
& \hat{C}_{m, \hat{k}}=\sum_{l=0}^{2 L-1} C_{m, l} \exp \left(-i \frac{\pi}{L} l k\right) \quad(k=0, \ldots, 2 L-1), \\
& \hat{G}_{m, \hat{k}}=\sum_{l=0}^{2 L-1} G_{m, l} \exp \left(-i \frac{\pi}{L} l k\right) \quad(k=0, \ldots, 2 L-1) .
\end{aligned}
$$

The DFT of $\phi_{0, l}^{m}$ can be expressed as

$$
\hat{\phi}_{0, \hat{k}}^{m}=\hat{C}_{m, \hat{k}} \hat{G}_{m, \hat{k}} \quad(0 \leq k \leq 2 L-1) .
$$

The solution of $\phi_{0, l}^{m}$ is obtained by the inverse DFT

$$
\phi_{0, l}^{m}=\frac{1}{2 L} \sum_{k=0}^{2 L-1} \hat{\phi}_{0, \hat{k}}^{m} \exp \left(i \frac{\pi}{L} l k\right) \quad(0 \leq l \leq 2 L-1) .
$$

Technically, we compute $\phi_{0, l}^{m}$ in Eq. (17) by 3 times FFT instead of computing it directly by Eq. (18). Since the dimension of mesh and the grid number are both constant during the course of tracking, $G_{j, l}$ is constant and only 2 times DFT is needed in fact. The potentials on other boundaries can be computed by a similar method.

After the boundary potentials are known, the problem is converted into a Dirichlet one of the Poisson equation. The five-point difference scheme is used to approximate the two-dimensional Laplacian operator

$$
\frac{\phi_{j-1, l}+\phi_{j+1, l}-2 \phi_{j, l}}{h_{x}^{2}}+\frac{\phi_{j, l-1}+\phi_{j, l+1}-2 \phi_{j, l}}{h_{y}^{2}}=-\frac{\rho_{j, l}}{\epsilon_{0}} .
$$

The Fourier analysis and cyclic reduction (FACR) method is used to solve Eq. (26), then we can obtain the potentials on the inside grid points.

The field strength $\vec{E}=-\nabla \phi$ on a grid point is computed using the six-point difference scheme. The field not on the grid points is computed using the same smoothing scheme as that used in the charge assignment.

The Bassetti-Erskine formula [11] can be used to compute the field generated by a two-dimensional Gaussian beam. The filed solver code has been checked against the theoretical values, and the coincidence is fine $[9,12]$.

\section{Finite bunch length and parallel scheme}

The bunch length effect is included by longitudinal slices. As a shorter (or zero length) bunch, a slice interacts in turn with the ones in the opposite beam. The interaction between two slices can be treated using the method described in the previous section. The longitudinal boundaries of slices are chosen so that the number of macroparticles in each slice is uniform.

Because of the synchrotron oscillation, the $z$ value of a particle varies each turn. That is to say the particles in one 
slice may enter the others of the same bunch. For high energy electron or positron storage ring, the synchrotron tune is generally very small (in the order of $10^{-2}$ ), that is to say the oscillation frequency is very low. It is reasonable for us to assume that it is impossible for a particle in one slice to jump into not-adjacent ones the next turn when the slice number is not very large.

When the finite bunch length effect is taken into account, the computing time increases linearly by a factor of $n^{2}$, where $n$ is the number of slices in one bunch. A supercomputer is necessary for the three-dimensional beambeam simulation.

The standard message passing interface (MPI) is used to parallelize the code. It is natural to represent one slice with one MPI node: the macroparticles' data in a slice is stored in the corresponding node. When two slices collide, their corresponding nodes assign the charges of macroparticles to grid points, respectively, exchanging the smoothened charge distribution on grid, and then the beam-beam force experienced by a slice can be computed by its corresponding node. Since a slice needs to exchange macroparticles with the adjacent ones, the computing time increases linearly by a factor of $(n+1)$. The parallel scheme is shown in Fig. 1. The scheme is not scalable as Qiang's [13], while it is efficient and suitable for small or medium-scale computer clusters.

\section{Interpolation of field}

We have taken the finite bunch length effect into account by dividing the beam into longitudinal slices, while the convergence of the slice number is not good. Following Ohmi's idea [14], an interpolation of beam-beam potential is done when the collision between two slices is considered. The required number of slices can be dramatically reduced.

The scheme is shown in Fig. 2. Slice $i$ in a bunch collides with slice $j$ in the opposite bunch. Here let us consider the beam-beam force experienced by $i$ and generated by $j$. The two-dimensional potential is computed at the longitudinal positions $s_{f}$ and $s_{b}$. Here $s_{f}$ is the point where the front end $z_{i, f}$ of $i$ meets the center $z_{j}$ of $j$, and $s_{b}$ is the point where the back end $z_{i, b}$ meets the center:

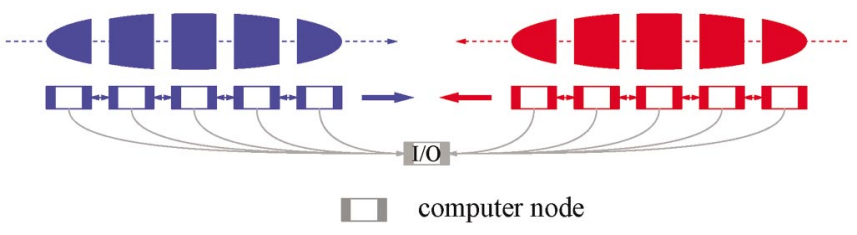

FIG. 1. (Color) Parallel scheme of SBBE. One MPI node is used to represent one slice. A slice exchanges macroparticles with the adjacent ones at IP, and then collides in turn with the ones in the other bunch. The I/O node is used to collect data and save them to a file. The computing time increases linearly by a factor of $(n+1)$, where $n$ is the number of slice in one bunch.

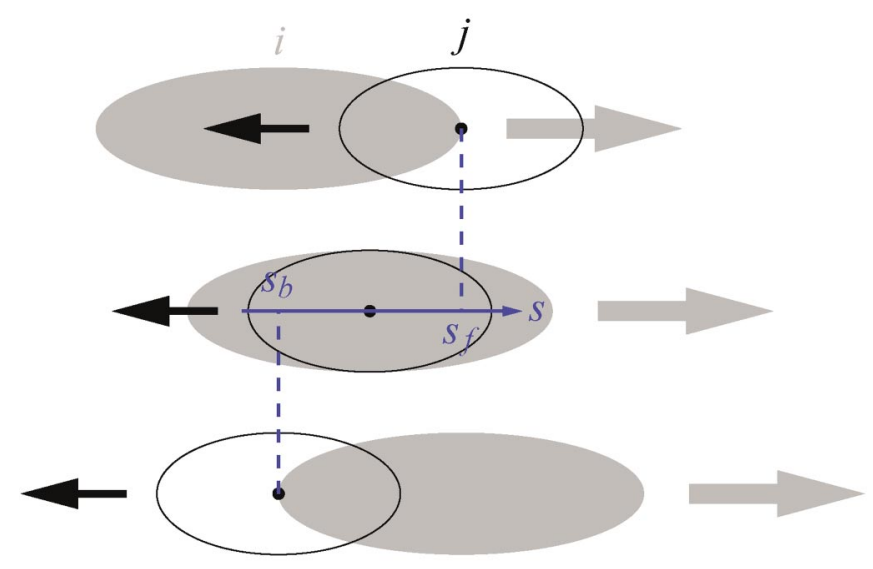

FIG. 2. (Color) Interpolation scheme of field. The beam-beam force experienced by slice $i$ and generated by slice $j$ is considered. The potential by $j$ is computed twice at $s_{f}$ and $s_{b}$. The point $s_{f}$ is the position where the front end of $i$ meets the center of $j$, and $s_{b}$ the position where the back end meets the center. The potential by $j$ at $s\left(s_{b} \leq s \leq s_{f}\right)$ can be calculated by linear interpolation.

$$
s_{f}=\frac{z_{i, f}-z_{j}}{2}, \quad s_{b}=\frac{z_{i, b}-z_{j}}{2} .
$$

The $s$ axes of the two beams coincide with opposite directions. Both $s_{f}$ and $s_{b}$ are represented in the $s$ axis of the slice $i$. The potential at $s\left(s_{b} \leq s \leq s_{f}\right)$ can be calculated by linear interpolation

$$
\phi(s)=\phi\left(s_{b}\right)+\frac{\phi\left(s_{f}\right)-\phi\left(s_{b}\right)}{s_{f}-s_{b}}\left(s-s_{b}\right) .
$$

A particle in slice $i$ meets the center of slice $j$ at $s$. The beam-beam force experienced by the particle is computed with following steps: (a) it is drifted to the longitudinal position $s$ and TSC weights on nine nearest points are calculated; (b) the potentials on twenty-five nearest points are calculated by interpolation; (c) the transverse field strength on nine nearest points is calculated by six-point difference scheme; (d) the field experienced by the particle is calculated by using the weighted summation of the fields at nine nearest points with the TSC weight.

\section{E. Horizontal crossing angle}

When there exists a crossing angle during collision, the beam-beam force generated by the opposite beam is not in the transverse direction of the beam that is experiencing the force. In this case the force depends not only on the transverse position but also on the longitudinal position. This makes it much more difficult to treat the beam-beam interaction with a finite crossing angle.

However, we can find a new frame where the $s$ axes of the two colliding beams coincide with opposite directions. The new frame is called the head-on frame which moves with a speed $c \sin \theta$ relative to the laboratory frame and in 
the direction of angle bisector of the two old $s$ axes. Here $\theta$ is the half crossing angle. The Lorentz transformation is used for the conversion from the laboratory frame to the head-on frame. We still employ the accelerator coordinate $\mathbf{x}$. The transformation $\mathcal{M}_{L}$ is given by [15]

$$
\begin{aligned}
& p_{x}^{*}=\frac{p_{x}-h \tan \theta}{\cos \theta}, \quad p_{y}^{*}=\frac{p_{y}}{\cos \theta}, \\
& p_{z}^{*}=p_{z}-p_{x} \tan \theta+h \tan ^{2} \theta, \quad h^{*}=\frac{h}{\cos ^{2} \theta}, \\
& x^{*}=x\left(1+\frac{\partial h^{*}}{\partial p_{x}^{*}} \sin \theta\right)+z \tan \theta, \quad y^{*}=y+x \frac{\partial h^{*}}{\partial p_{y}^{*}} \sin \theta, \\
& z^{*}=\frac{z}{\cos \theta}+x \frac{\partial h^{*}}{\partial p_{z}^{*}} \sin \theta,
\end{aligned}
$$

where

$$
h=\left(1+p_{z}\right)-\sqrt{\left(1+p_{z}\right)^{2}-p_{x}^{2}-p_{y}^{2}} .
$$

An asterisk here designates a dynamical variable in the head-on frame. Note that the $x^{*}$ and $y^{*}$ axes are defined in the same direction for both beams, while the $s^{*}$ axis is defined in opposite directions. After the transformation $\mathcal{M}_{L}$, the collision is treated as a head-on collision, and then with the inverse transformation $\mathcal{M}_{L^{-1}}$ we obtain the dynamical variable in the laboratory frame after collision.

We notice that the half crossing angle $\theta$ in Eq. (29) is a signed value. If the angle between axes $s$ and $x^{*}$ is larger than $90^{\circ}$, the value is negative, and if the angle is smaller than $90^{\circ}$, the value is positive, as shown in Fig. 3. The angle sign is minus for BEPCII, while the results show that the sign makes no difference. The sign takes effect in more sophisticated cases.

The transformation $\mathcal{M}_{L}$ which is called "Lorentz boost" by Hirata is not symplectic, and it was pointed out by Hirata [15] and Ohmi [14] that the whole beambeam map is symplectic if $\mathcal{M}_{L^{-1}}$ is used after the beambeam kick. Cai [16] introduced a geometrical method to

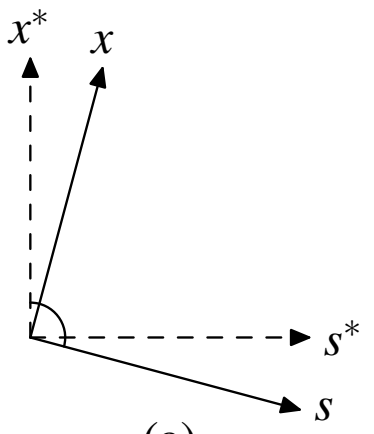

(a)

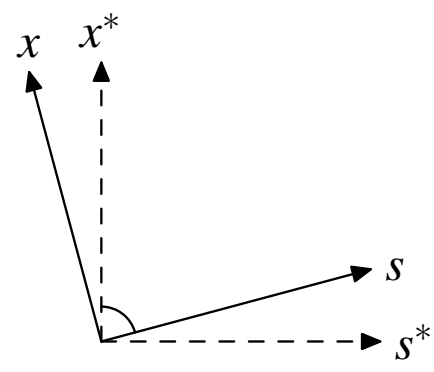

(b)
FIG. 3. Sign of the half crossing angle in $\mathcal{M}_{L}$. The sign is minus if the angle between the two axes $s$ and $x^{*}$ is larger than $90^{\circ}$, which is shown in (a). The sign is plus if the angle between the two axes $s$ and $x^{*}$ is less than $90^{\circ}$, which is shown in (b). treat exactly a collision without using of the Lorentz boost for a finite crossing angle, where the symplecticity is preserved throughout the collision process.

The loss in luminosity due to geometrical (hourglass) effect for Gaussian beams is $R$, and the reduction factor coming from a nonzero horizontal crossing angle is $R_{L}$ [17]. The two factors are both obtained without beambeam interaction. The code has been checked against the theoretical values by calculating the luminosities at low beam currents $\left(\xi_{y}<0.004\right)$ with or without a crossing angle, and the difference is less than $1 \%$ [12].

\section{SIMULATION RESULTS}

The code has been benchmarked with Ohmi's [14], and the difference in simulated luminosity between the two codes is less than $10 \%$.

We studied the beam-beam effects in BEPCII by simulation using SBBE. The results are shown in the following sections. The design parameters of BEPCII is shown in Table I. The design luminosity is $1.0 \times 10^{33} \mathrm{~cm}^{-2} \mathrm{~s}^{-1}$ at $1.89 \mathrm{GeV}$.

\section{A. Choice of model parameters}

There are some nonphysical parameters in the model of our simulation code, such as the number of the longitudinal slices per bunch, the number of the macroparticles per bunch, and the dimension of mesh and grid number. We call them model parameters. The choice of these parameters will impact the precision of the simulation result. Since it is difficult to describe the influence quantitatively, we determine the parameters empirically and numerically. The choice is a compromise between the computing time and the precision of result. The model parameters used in our simulation are shown in Table II. In the following we show how to determine these parameters and the results vary with them.

TABLE I. Design parameters of BEPCII.

\begin{tabular}{lcc}
\hline \hline$E$ & Beam energy & $1.89 \mathrm{GeV}$ \\
$C$ & Circumference & $237.53 \mathrm{~m}$ \\
$N_{b}$ & Bunch number & 93 \\
$I_{b}$ & Bunch current & $9.8 \mathrm{~mA}$ \\
$\xi_{y}$ & Beam-beam parameter & 0.04 \\
$\theta_{c}$ & Horizontal crossing angle & $2 \times 11 \mathrm{mrad}$ \\
$\beta_{x}^{*} / \beta_{y}^{*}$ & Beta function at IP & $1 \mathrm{~m} / 1.5 \mathrm{~cm}$ \\
$\epsilon_{x} / \epsilon_{y}$ & Transverse emittance & $144 \mathrm{~nm} / 2.2 \mathrm{~nm}$ \\
$\sigma_{z}$ & Bunch length & $1.5 \mathrm{~cm}$ \\
$\sigma_{e}$ & Energy spread & $5.16 \times 10^{-4}$ \\
$\nu_{x} / \nu_{y}$ & Betatron tune & $6.53 / 7.58$ \\
$\nu_{s}$ & Synchrotron tune & 0.034 \\
$\tau_{x} / \tau_{y}$ & Transverse damping time & $31553 \mathrm{turn} / 31553 \mathrm{turn}$ \\
$\tau_{s}$ & Synchrotron damping time & $15777 \mathrm{turn}$ \\
\hline \hline
\end{tabular}


TABLE II. Model parameters.

\begin{tabular}{lc}
\hline \hline Slice number/bunch & 5 \\
Macroparticles number/bunch & $30 \times 10^{4}$ \\
Mesh size & $30 \sigma_{x} \times 60 \sigma_{y}$ \\
Grid number & $128 \times 256$ \\
\hline \hline
\end{tabular}

\section{Slice number}

The more the slice number, the more exact the simulation result. Since the computing time increases linearly with $(n+1)$, a suitable slice number $n$ is necessary. The required number depends on the beam-beam parameter $\xi_{y}$. The beam distribution is deformed during the process of collision. It is an oscillation angle of a particle in the potential of the opposite beam that characterizes the deformation [14]

$$
\chi=\sqrt{\frac{4 \pi \xi_{y} \sigma_{z}}{\beta_{y}}} .
$$

The oscillation angle is divided by the times of collision with slices. The angle in each collision should be smaller than 1. Figure 4 shows the evolution of simulated luminosity for various slice number. The difference in the final luminosity is less than $2 \%$ between 5 and 13 slices, and less than $1 \%$ between 9 and 13. It is $\chi \approx 0.7 \sim 1.0$ for our parameter region, $\xi_{y}=0.04 \sim 0.08$ and $\sigma_{z} \simeq \beta_{y}$. In this paper, simulation results are obtained with 5 slices, then the divided angle is $0.14 \sim 0.2$ for each slice.

\section{Macroparticle number}

There are $\sim 10^{10}$ particles in one bunch, which is impossible for us to allocate so much memory space to store all the particles' data in the simulation. Macroparticles are used to represent the distribution of a beam. Since the PIC

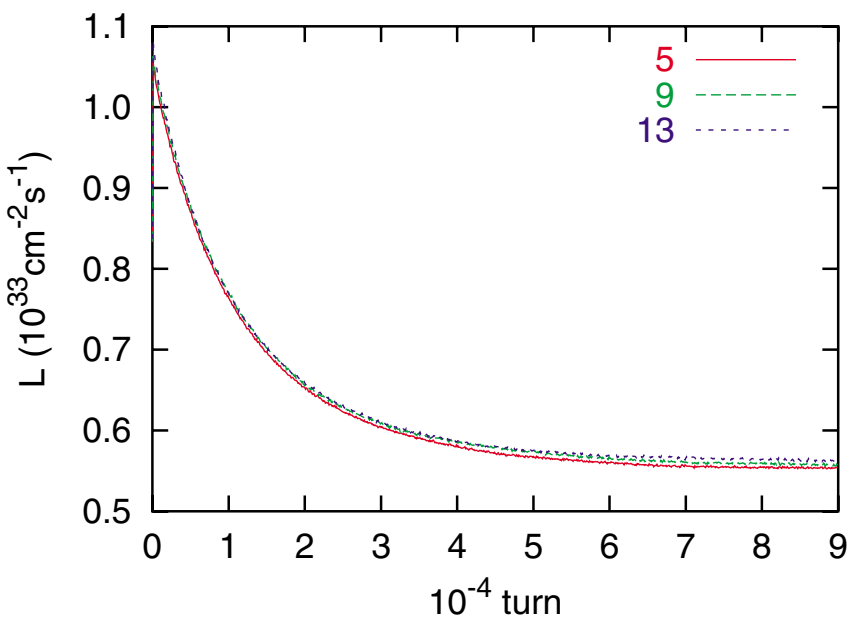

FIG. 4. (Color) Evolution of luminosity for various slice numbers. method is used to calculate the potential, in the case that mesh dimension and grid number are fixed, the accuracy will not improve any more with the increase of macroparticle number when it is larger than some threshold value. Figure 5 shows the evolution of simulated luminosity for various macroparticle number. The difference in the final luminosity is less than $2 \%$ between $15 \times 10^{4}$ and $60 \times 10^{4}$, and less than $1 \%$ between $30 \times 10^{4}$ and $60 \times$ $10^{4}$. In this paper, simulation results are obtained with $30 \times 10^{4}$ macroparticles.

\section{Mesh size and grid number}

The macroparticles outside the area covered by the mesh are supposed to lose in our code. The loss ratio is less than $10^{-4}$ with the mesh area $30 \sigma_{x} \times 60 \sigma_{y}$ after 3 times transverse damping time. It shows that the dimension of mesh is large enough to contain the bunch core and we do not try any other choices.

In order to achieve the required resolution, a few grid points per $\sigma$ of the beam are needed, otherwise the size of grid is too large for numerical computation. For the mesh area of $30 \sigma_{x} \times 60 \sigma_{y}$ and grid number of $128 \times 256$, there are 4 grid points per $\sigma_{x}$ and 4 grid points per $\sigma_{y}$. Truncation errors in the Poisson solver are of order $h_{x}^{2}$ and $h_{y}^{2}$ [7]. Since the nominal beam aspect ratio is $66: 1$ at IP, truncation errors in the horizontal plane are dominant. Figure 6 shows the evolution of luminosity for various grid number. The difference in the final luminosity is less than $6 \%$ between $128 \times 256$ and $512 \times 256$, and less than $2 \%$ between $256 \times 256$ and $512 \times 256$. The result is nearly the same for $256 \times 256$ and $256 \times 512$. That is to say the result is mainly affected by the dimension of $h_{x}$. In order to save the computing time, $128 \times 256$ is used in our simulation.

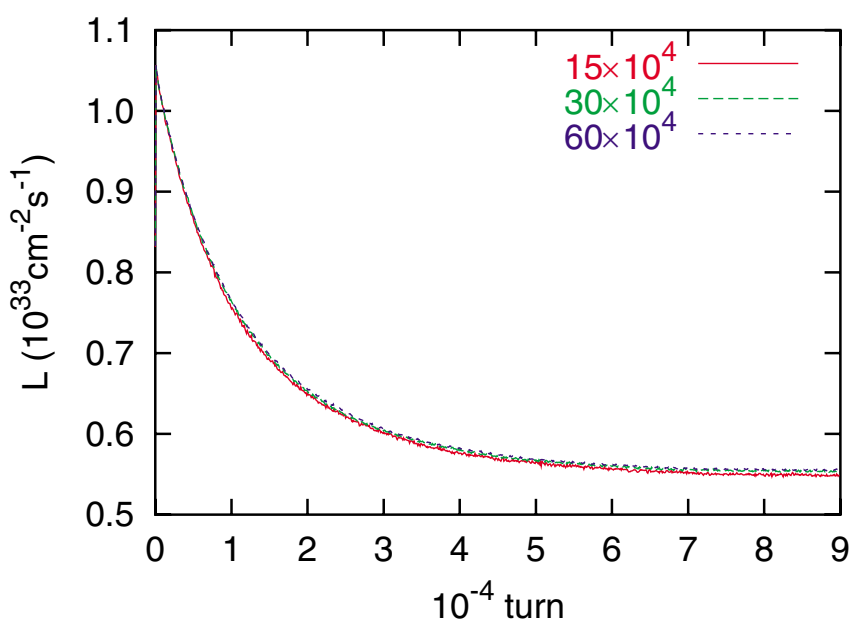

FIG. 5. (Color) Evolution of simulated luminosity for various macroparticle numbers. 


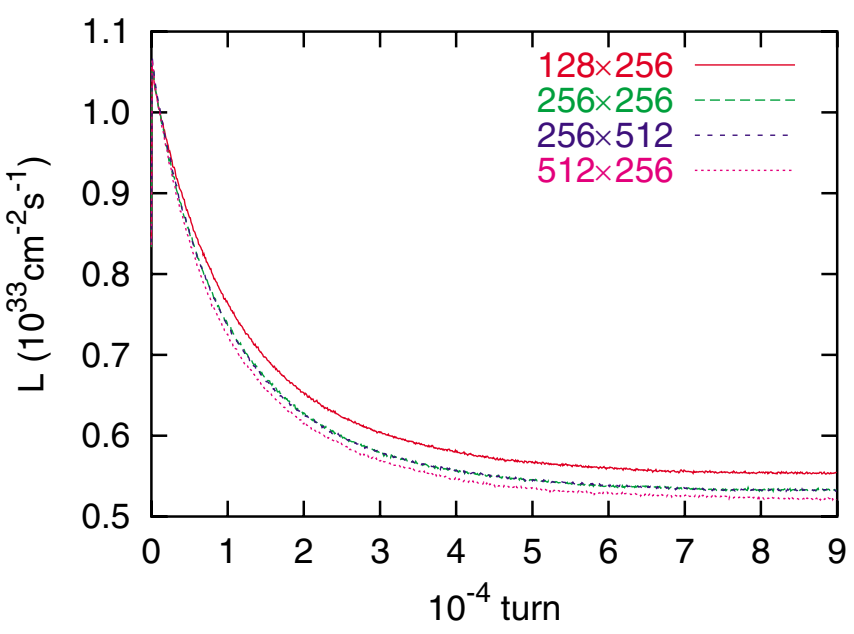

FIG. 6. (Color) Evolution of simulated luminosity for various grid numbers.

\section{B. Tune survey}

It is well known that the working points strongly affect the beam-beam interaction. Here we investigate how the tunes affect the luminosity and try to find the best area in the tune space. Empirically, the strong-strong simulation was done in the area of $0.505 \leq \nu_{x} \leq 0.545$ and $0.55 \leq$ $\nu_{y} \leq 0.595$ with a step of 0.005 .

Figure 7 shows the contour plot of luminosity and beam size versus tune. The best area is near $(0.505,0.57)$ where the luminosity is about $80 \%$ of the design value. In the survey area, the horizontal size is mainly affected by the horizontal tune, and its minimum area is near $\nu_{x}=0.535$.

No clear vertical coherent motion is seen in the tune space. Horizontal coherent motions in $\langle x\rangle$ are mainly decided by horizontal tunes. Figure 8 shows the evolution of the horizontal dipole amplitude for various horizontal tunes. The amplitude of coherent oscillation in $\langle x\rangle$ is $\sim 100 \mu \mathrm{m}$ at $\nu_{x}=0.515$, nearly one third of the nominal beam size. The coherent motion may cause instability or influence the beam life time in the real machine.
Since the design working point is not in the best area according to our survey, we will study the beam-beam effects not only at the design tune but also at another point $(0.51,0.57)$ in the following sections.

\section{Beam-beam limit}

Colliders are generally designed to operate with beam current before the saturation of the beam-beam parameter. Here the parameter is calculated using the luminosity as follows

$$
\xi_{y}=\frac{2 r_{e} \beta_{y}}{N \gamma} \frac{L}{f_{\text {rep }}} .
$$

The saturation phenomenon is called the beam-beam limit, which means that the luminosity varies linearly with the bunch current. Further increasing of the current results in development of beam halo, leading to low beam lifetime. This ultimately limits the maximum beam current that can safely collide. Here the simulations are carried out for various bunch populations to obtain the beam-beam limit.

\section{1. @ $(0.53,0.58)$}

Figure 9 shows the simulation results for various bunch currents at the design working point. As seen in the figure, $\xi_{y}$ varies linearly with current when the latter is less than $10 \mathrm{~mA}$, reaches maximum $(\sim 0.025)$ near $13 \mathrm{~mA}$, and tapers when the current is further increased. The fluctuation of $\xi_{y}$ is reflected by the bunch size when the current is in the range of [4 mA, $8 \mathrm{~mA}]$. When the current is more than $14 \mathrm{~mA}$, further increasing results in the sharp rise of particle loss. There is no clear coherent motion at the design working point.

\section{2. @(0.51,0.57)}

Figure 10 shows the simulation results for various bunch currents at $\left(\nu_{x}, \nu_{y}\right)=(0.51,0.57)$. As seen in the figure, $\xi_{y}$ varies linearly with current when the latter is less than

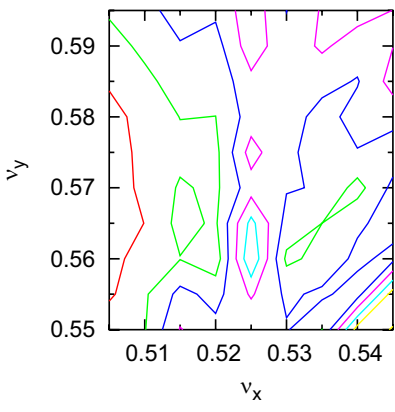

(a)

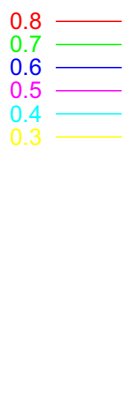

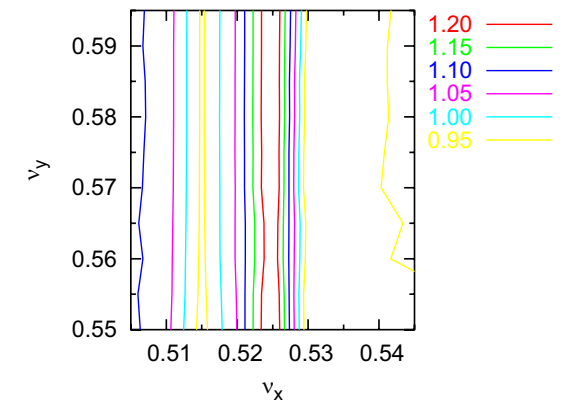

(b)

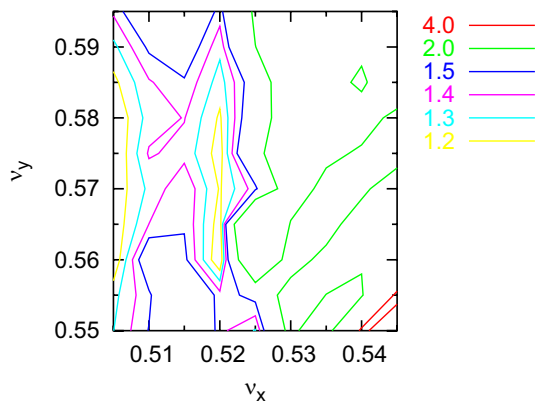

(c)

FIG. 7. (Color) Luminosity and beam size (rms) versus transverse tune at design parameters. The luminosity, which is depicted in (a), is normalized by the design value. The horizontal and vertical beam sizes, which are normalized by the corresponding nominal values, are depicted in (b) and (c), respectively. 

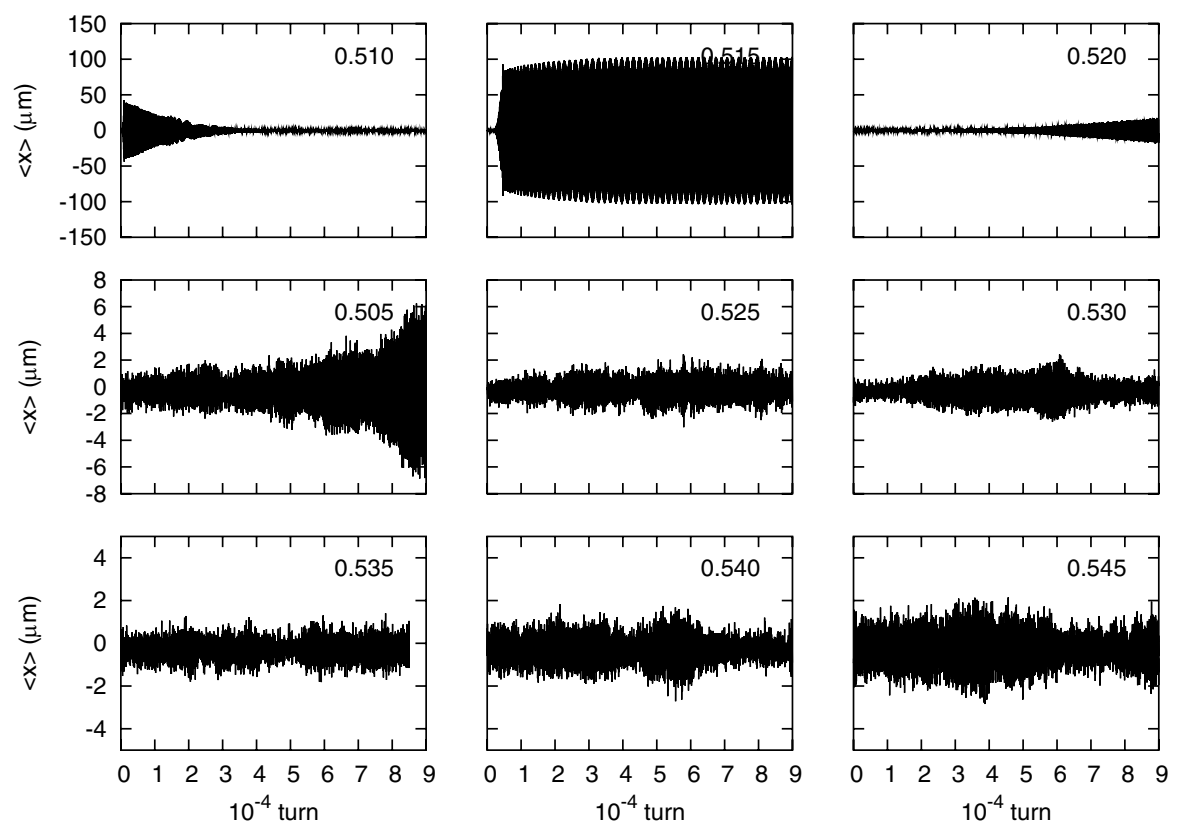

FIG. 8. Evolution of the horizontal dipole amplitude of the beam. The corresponding horizontal tune is written in each figure. The vertical tune is 0.57 .

$10 \mathrm{~mA}$, reaches maximum $(\sim 0.035)$ near $12 \mathrm{~mA}$, and tapers when the current is further increased. When the current is more than $15 \mathrm{~mA}$, the two colliding beams that start out with equal sizes become very unequal: one of the beams gets blown up transversely to a very large size, while the other remains small. There also exist asymmetry in particle loss between two beams at a high current. There
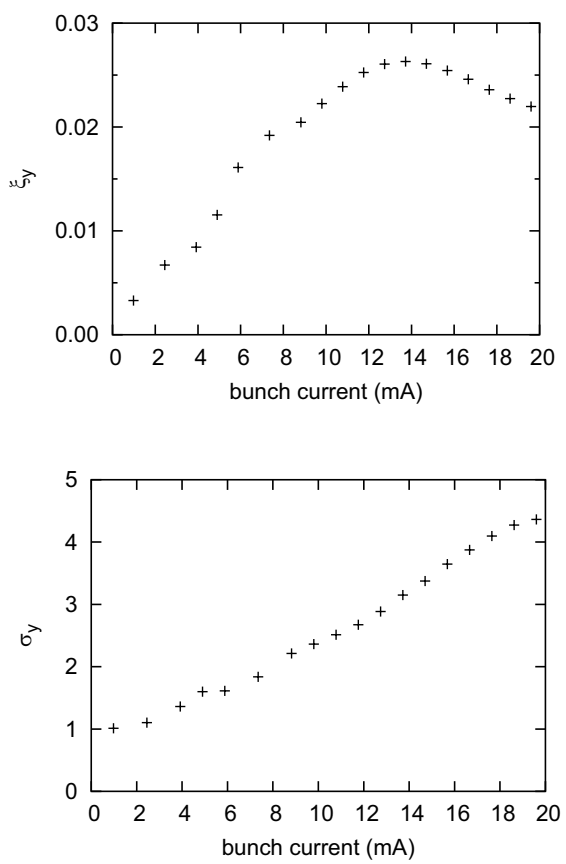

is no clear coherent motion in $\langle y\rangle$. Figure 11 shows the evolution of $\langle x\rangle$ for various bunch currents. It is strange that the coherent oscillation happens when the current $I_{b} \in$ [2.94 mA, $4.90 \mathrm{~mA}$ ], while it disappears at a higher current $I_{b} \in[5.88 \mathrm{~mA}, 7.84 \mathrm{~mA}]$. The coherent motion is damped near $I_{b}=9.8 \mathrm{~mA}$ after several ten thousand turns, and not damped when $I_{b}>12 \mathrm{~mA}$.
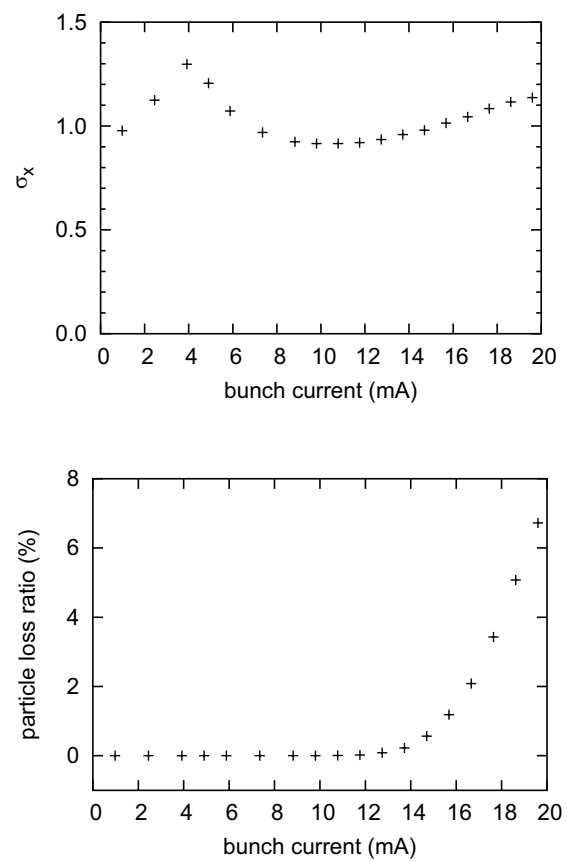

FIG. 9. Beam-beam parameters, beam sizes (rms), and macroparticle loss versus bunch current at $\left(\nu_{x}, \nu_{y}\right)=(0.53$, 0.58). The size is normalized by the nominal value without beam-beam interaction. There is no clear difference between two colliding beams. The result is given after 150000 turns. 

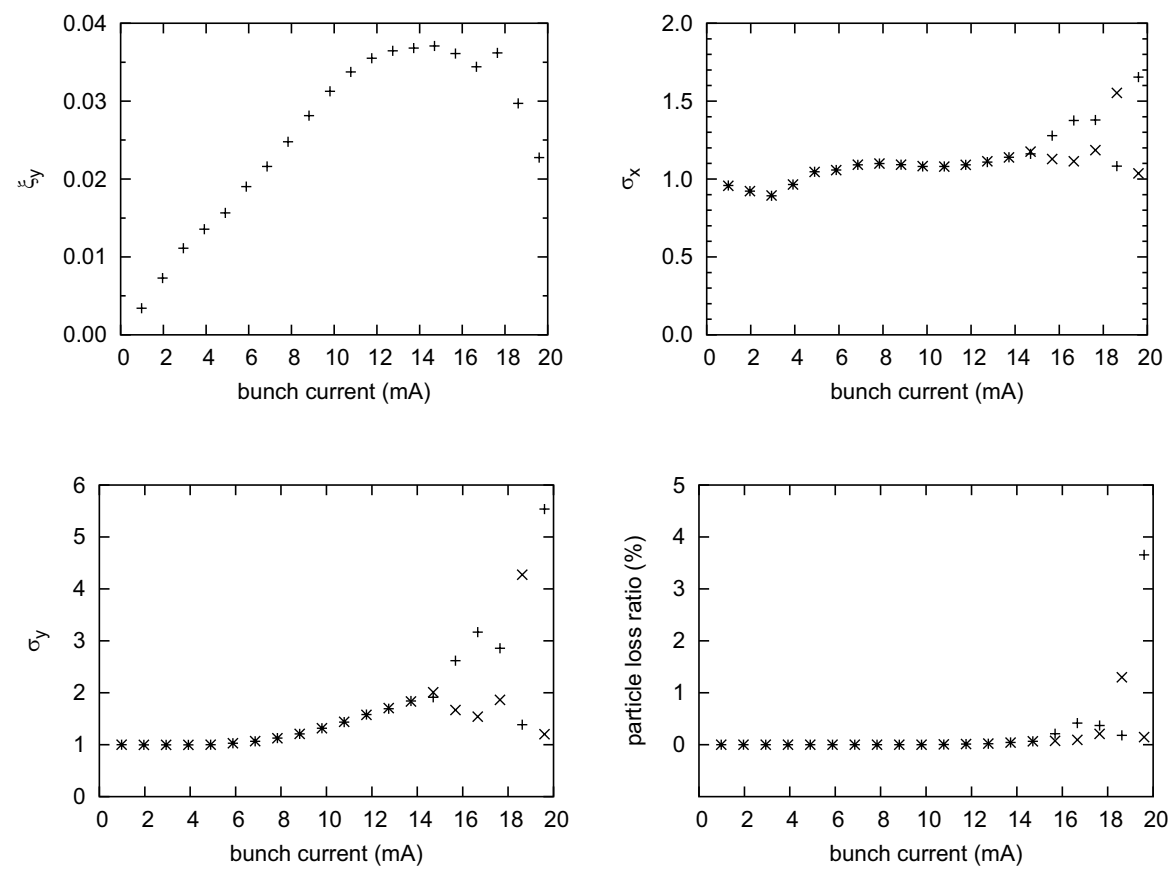

FIG. 10. Beam-beam parameters, beam sizes (rms), and macroparticles loss versus bunch current at $\left(\nu_{x}, \nu_{y}\right)=(0.51,0.57)$. The size is normalized by the nominal value without beam-beam interaction. The signs $\times$ and + indicate two colliding beams whose nominal parameters are identical in BEPCII. The result is given after 150000 turns.
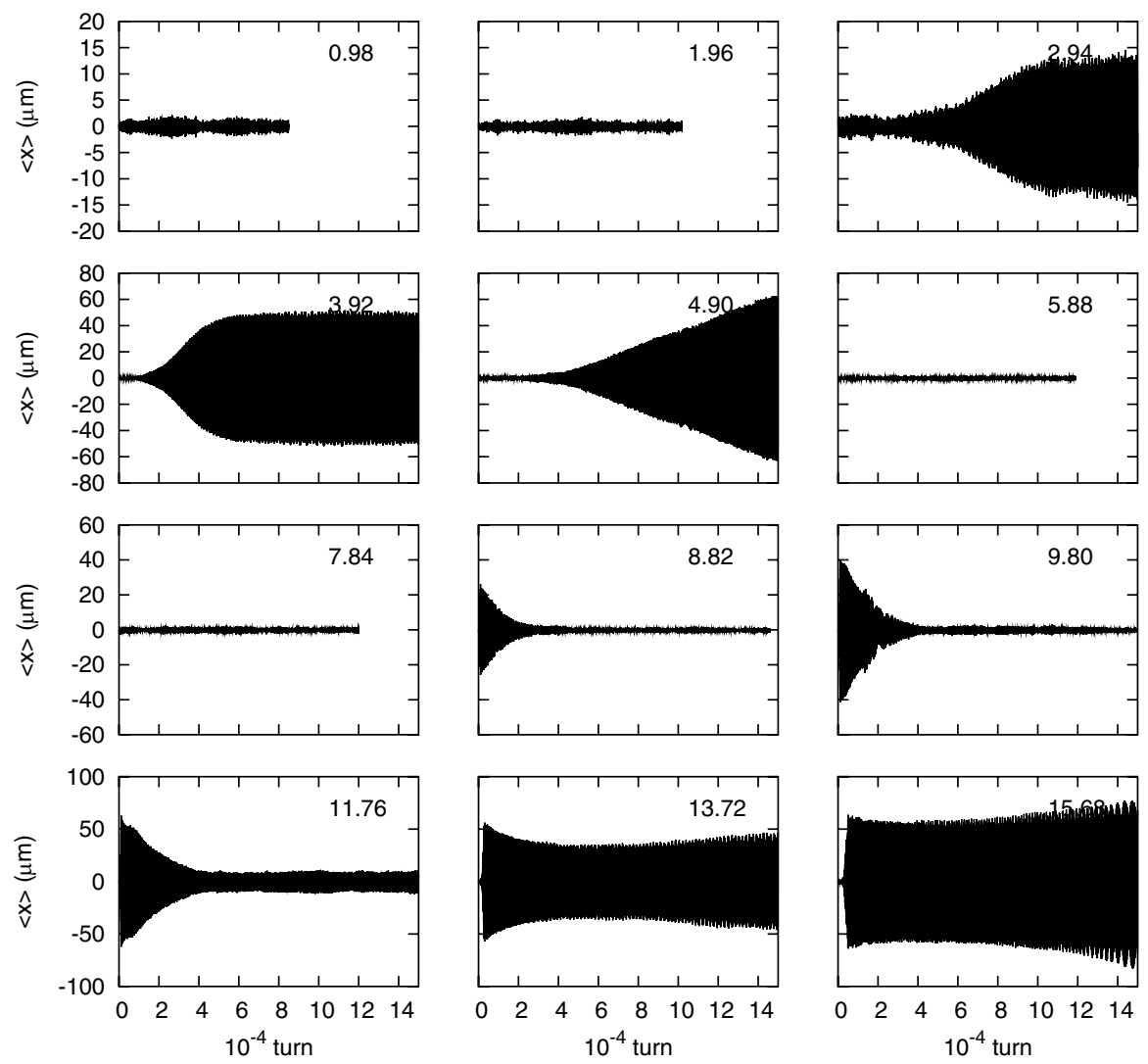

FIG. 11. Evolution of the horizontal dipole amplitude of the beam at $\left(\nu_{x}, \nu_{y}\right)=(0.51,0.57)$. The corresponding bunch current (unit: $\mathrm{mA}$ ) is written in each figure. The amplitude reaches steady state after 150000 turns for $I_{b}=4.90 \mathrm{~mA}$. 

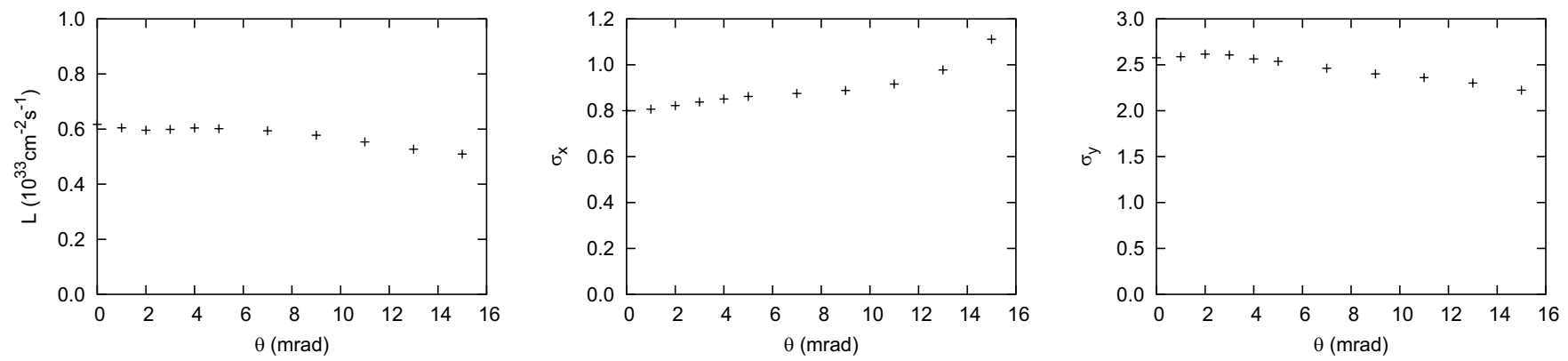

FIG. 12. Luminosity and beam size versus crossing angle at $\left(\nu_{x}, \nu_{y}\right)=(0.53,0.58)$. The abscissa is half of the horizontal crossing angle. The luminosity is the bunch value multiplied with 93 (bunch number). The size is normalized by the nominal value without beam-beam interaction.

\section{Crossing angle dependence}

The simulations for various crossing angles were performed in order to study the effect.

\section{1. @(0.53,0.58)}

The luminosity and beam size versus crossing angle at the design working point is shown in Fig. 12. The luminosity degradation due to a small angle $(\theta \lesssim 5 \mathrm{mrad})$ is very little. And we need not worry about the effect of a large crossing angle, the degradation due to $2 \times 11 \mathrm{mrad}$ is less than $10 \%$. The horizontal beam size increases monotonically with the crossing angle. The vertical size remains nearly constant for a small crossing angle, and there is a gradual decrease for increasing angle. There is no clear coherent motion.

\section{2. @ $(0.51,0.57)$}

The luminosity and beam size versus crossing angle at $\left(\nu_{x}, \nu_{y}\right)=(0.51,0.57)$ is shown in Fig. 13. Here the dependence of luminosity on angle is stronger than that at the design tune. Compared with the head-on luminosity, it decreases by $\sim 17 \%$ for $\theta=5 \mathrm{mrad}, \sim 30 \%$ for $\theta=$ $11 \mathrm{mrad}$, and $\sim 42 \%$ for $\theta=15 \mathrm{mrad}$. The horizontal beam size increases monotonically with crossing angle. The fluctuation of vertical size is less than $10 \%$ for various angles. There is no clear coherent motion in $\langle y\rangle$. Figure 14 shows the evolution of $\langle x\rangle$ for various crossing angles. There exists coherent motion for $\theta \leq 5 \mathrm{mrad}$. The motion is damped for $\theta \leq 9 \mathrm{mrad}$, and disappears for $\theta \leq$ $15 \mathrm{mrad}$.

\section{DISCUSSION}

In order to study the beam-beam effects in BEPCII, we have developed a three-dimensional strong-strong code. The bunch length effect is included by using longitudinal slices and an interpolation scheme is employed to reduce the required slice number. The code is parallelized with MPI, and the computing time increases linearly with $(n+1)$ where $n$ is the slice number. The number of MPI nodes, $2 n+1$, is fixed. The two-dimensional beam-beam potential on mesh boundary is computed using an FFT method, and the potential inside is computed using the FACR method. The collision with a finite crossing angle is treated as a head-on collision by the Lorentz transformation.

Though the SBBE code is termed a three dimensional one, the actual potential calculation is only two dimensional. In the longitudinal dimension, the finite bunch length, not longitudinal field, is taken into account, while this is a good approximation for ultrarelativistic beams.
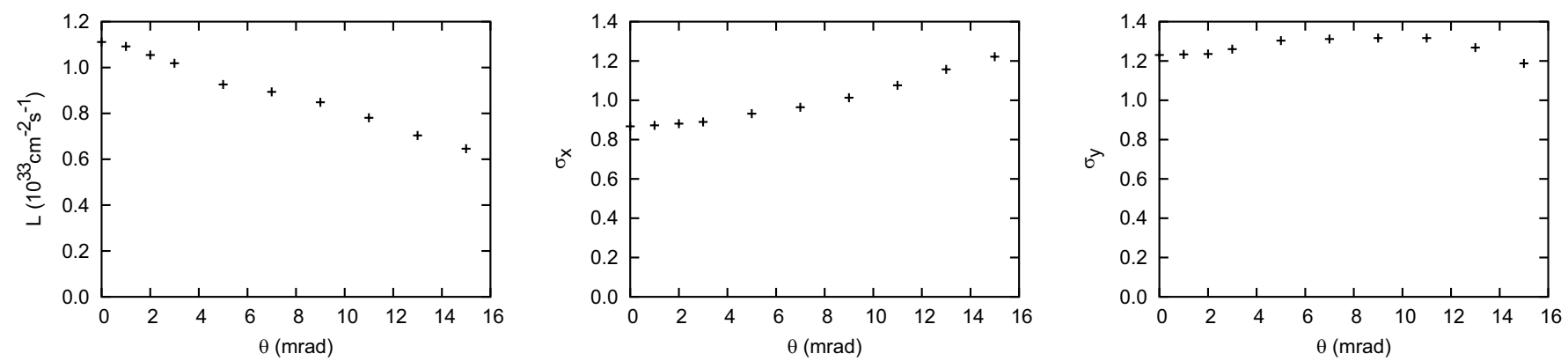

FIG. 13. Luminosity and beam size versus crossing angle at $\left(\nu_{x}, \nu_{y}\right)=(0.51,0.57)$. The abscissa is half of the horizontal crossing angle. The luminosity is the bunch value multiplied with 93 (bunch number). The size is normalized by the nominal value without beam-beam interaction. 

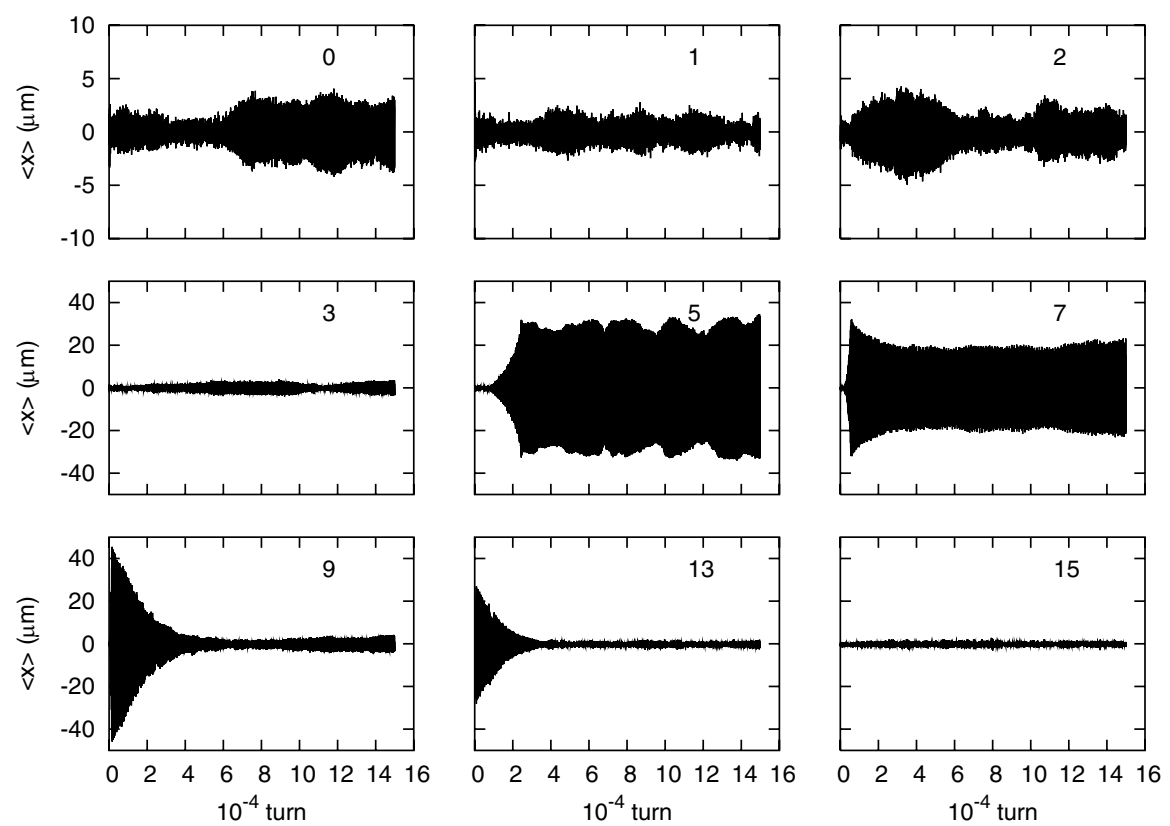

FIG. 14. Evolution of the horizontal dipole amplitude of the beam for various crossing angles at $\left(\nu_{x}, \nu_{y}\right)=(0.51,0.57)$. The corresponding half-crossing angle (unit: mrad) is written in each figure.

We have studied the beam-beam effects in BEPCII using the code. The result shows that the luminosity at the design tune is $\sim 50 \%$ of the design luminosity.

A tune survey has been done in the area $\left(\nu_{x}, \nu_{y}\right) \in$ $[0.505,0.545] \times[0.550,0.595]$ with a step of 0.005 . It seems that the best area is near $(0.505,0.57)$.

The beam-beam limit at $(0.53,0.58)$ (the design working point) has been studied, and the results show that $\xi_{y}$ reaches maximum, $\sim 0.025$, near $I_{b}=13 \mathrm{~mA}$. While at $(0.51,0.57)$, the beam-beam parameter saturates near $I_{b}=$ $12 \mathrm{~mA}, \sim 0.035$.

The crossing angle dependence in BEPCII is also studied. The luminosity degradation due to crossing angle is about $10 \%$ at $(0.53,0.58)$. The luminosity for head-on collision does not have any gain for that with crossing angle in KEKB at the working point [14]. The degradation is about $30 \%$ at $(0.51,0.57)$, where the crossing angle dependence is looser than KEKB [14]. This is perhaps due to that the design beam-beam parameter is 0.04 for BEPC, which is less than KEKB $(\sim 0.1)$.

It is concerned that if the horizontal coherent motion near $\nu_{x}=0.51$ would be damped in the real machine, otherwise it may cause instability and reduce the beam lifetime. The coherent motion is affected by the tune, bunch current, and crossing angle. In our simulations, the coherent motions are probably excited by two oscillation lines, $2 \nu_{x}-\nu_{s}=n\left(\nu_{x}=0.517\right)$ or $2 \nu_{x}-2 \nu_{s}=n\left(\nu_{x}=\right.$ $0.534)$. In Figs. 8,11 , and $14, \nu_{x, \pi} \gtrsim 0.517$ or 0.534 when there exists coherent motion, $\nu_{x, \pi} \leqslant 0.517$ or 0.534 when the oscillation is damped, otherwise it is far away from the two oscillation points. It seems that the coherent motion would be excited if $\nu_{x, \pi}$ is too near an oscillation point. In
Fig. 8, $\nu_{x, \pi}$ increases and passes through the two oscillation points 0.517 and 0.534 during the course of increasing the horizontal tune. In Fig. 11, $\nu_{x, \pi}$ increases and passes through the two oscillation points 0.517 and 0.534 during the course of increasing the bunch current. In Fig. 14, $\nu_{x, \pi}$ reduces and passes through the oscillation point 0.534 during the course of increasing the crossing angle. Further study on this topic needs to be done in the future.

In this paper, simulations for BEPCII have been done with single bunch in each beam, but BEPCII is designed for 93 bunches per beam. The parasitic beam-beam effect was estimated by Tennyson's formula [18] in [19], which shows that the incoherent tune shift $\delta \nu_{y}$ experienced by a particle at the center of the bunch and due to parasitic crossings is in the order of $10^{-3}$. It seems that long-range beam-beam effects would not be very important in BEPCII.

\section{ACKNOWLEDGMENTS}

The first author would like to thank members of accelerator physics group in IHEP. This work was supported by the Chinese National Foundation of Natural Sciences, Contract No. 10275079, JSPS Core University Program and Supercomputing Center, CNIC, CAS.

[1] Design Report of Bepcii-Accelerator Part [2003 (unpublished)], 2nd ed.

[2] K. Hirata, H. Moshammer, and F. Ruggiero, Part. Accel. 40, 205 (1993). 
[3] S. Krishnagopal and R. Siemann, Phys. Rev. Lett. 67, 2461 (1991).

[4] S. Krishnagopal, Phys. Rev. Lett. 76, 235 (1996).

[5] S. Krishnagopal, Phys. Rev. ST Accel. Beams 3, 024401 (2000).

[6] K. Ohmi, Phys. Rev. E 62, 7287 (2000).

[7] Y. Cai, A. W. Chao, S. I. Tzenov, and T. Tajima, Phys. Rev. ST Accel. Beams 4, 011001 (2001).

[8] J.T. Rogers, in Beam-Halo Dynamics, Diagnostics, and Collimation: 29th ICFA Advanced Beam Dynamics Workshop on Beam-Halo Dynamics, Diagnostics, and Collimation and the Beam-Beam'03 Workshop, AIP Conf. Proc. No. 693 (AIP, New York, 2003), Vol. 693, pp. 273-277.

[9] Y. Zhang, High Energy Phys. Nucl. Phys. 28, 1116 (2004), in Chinese.
[10] K. Hirata, CERN SL Note 97-57 AP, 1997.

[11] M. Bassetti and G. Erskine, CERN-ISR-TH/80-06, 1980.

[12] Y. Zhang, Ph.D. thesis, Institute of High Energy Physics, CAS, 2005, in Chinese.

[13] J. Qiang, M. A. Furman, and R. D. Ryne, J. Comput. Phys. 198, 278 (2004).

[14] K. Ohmi, M. Tawada, Y. Cai, S. Kamada, K. Oide, and J. Qiang, Phys. Rev. ST Accel. Beams 7, 104401 (2004).

[15] K. Hirata, Phys. Rev. Lett. 74, 2228 (1995).

[16] Y. Cai, ICFA Beam Dynamics Newsletter 34, 11 (2004).

[17] Handbook of Accelerator Physics and Engineering, edited by A.W. Chao and M. Tigner (World Scientific, Singapore, 1998), p. 248.

[18] J. Tennyson, ABC Note 28, 1991.

[19] S. Wang and Y. Cai, in Proceedings of the Particle Acceleration Conference, Knoxville, TN, 2005 (PAC'05). 